2006

\title{
Spatiotemporal dynamics of networks of excitable nodes
}

Aaron J. Steele

Mark Tinsley

Kenneth Showalter

Follow this and additional works at: https://researchrepository.wvu.edu/faculty_publications

\section{Digital Commons Citation}

Steele, Aaron J.; Tinsley, Mark; and Showalter, Kenneth, "Spatiotemporal dynamics of networks of excitable nodes" (2006). Faculty Scholarship. 337.

https://researchrepository.wvu.edu/faculty_publications/337

This Article is brought to you for free and open access by The Research Repository @ WVU. It has been accepted for inclusion in Faculty Scholarship by an authorized administrator of The Research Repository@ WVU. For more information, please contact ian.harmon@mail.wvu.edu. 


\title{
Spatiotemporal dynamics of networks of excitable nodes
}

\author{
Aaron J. Steele, Mark Tinsley, and Kenneth Showalter \\ Department of Chemistry, West Virginia University, Morgantown, West Virginia 26506-6045
}

(Received 27 October 2005; accepted 26 January 2006; published online 31 March 2006)

\begin{abstract}
A network of excitable nodes based on the photosensitive Belousov-Zhabotinsky reaction is studied in experiments and simulations. The addressable medium allows both local and nonlocal links between the nodes. The initial spread of excitation across the network as well as the asymptotic oscillatory behavior are described. Synchronization of the spatiotemporal dynamics occurs by entrainment to high-frequency network pacemakers formed by excitation loops. Analysis of the asymptotic behavior reveals that the dynamics of the network is governed by a subnetwork selected during the initial transient period. (c) 2006 American Institute of Physics.
\end{abstract}

[DOI: $10.1063 / 1.2177569$ ]

\begin{abstract}
Networks of excitable nodes with complex connectivity are common in the biological world, such as interacting neurons in brain tissue. We study networks of excitable nodes based on the photosensitive Belousov-Zhabotinsky (BZ) reaction, in which local and nonlocal links are possible. Reaction-diffusion waves propagating into neighboring regions comprise the local links, while the nonlocal links are created by nondiffusive jumps in the addressable excitable medium. The network initially develops by the spread of excitation through the medium via the local and nonlocal links, yielding insights into the growth of the network of excitable nodes, including the role played by path-length optimization. The dependence of the asymptotic spatiotemporal behavior on the number of nonlocal links is examined and described in terms of phase and frequency synchronization of the nodes. The dynamics of the excitable nodes leads to a pruning process that gives rise to a subnetwork of active links. The addressable excitable $\mathrm{BZ}$ medium allows the characterization of the growth and asymptotic dynamics of a network of excitable nodes.
\end{abstract}

\section{INTRODUCTION}

Recent developments in characterizing network architectures $^{1,2}$ have led to studies of more complex network systems, such as networks of coupled oscillators. ${ }^{3,4}$ These networks are natural models for a variety of biological and physical processes, including neural networks ${ }^{1,5-8}$ and networks of chemical oscillators. ${ }^{9}$ The effect of network architecture on the dynamics of coupled oscillators has been examined in studies of network synchronization. ${ }^{10,11}$ Recent studies have focused on the interrelations between network architecture and dynamical properties, such as weighted coupling strategies for improved synchronization within small world networks ${ }^{3}$ and the relationship between frequency locking to a pacemaker and the network depth. ${ }^{9}$

Networks of excitable nodes, with an excitable steady state being the locally stable state, are fundamentally different from networks with oscillatory nodes. Without some external perturbation, such a system will remain continually quiescent. A number of theoretical studies have been carried out with models of neurons as local excitable elements ${ }^{6,7,12,13}$ and with the susceptible-infected-susceptible (SIS) model for disease spreading. ${ }^{14}$ These studies include the characterization of synchronization ${ }^{6,14}$ as well as self-sustained oscillations arising from recursive feedback within a network. ${ }^{6}$ While networks of excitable nodes require an initial perturbation to exhibit complex dynamical behavior, once perturbed, such networks evolve to exhibit global oscillations that are similar to those exhibited in networks of oscillatory nodes. An important difference between networks of excitable and oscillatory nodes is that the excitable system evolves from the initial perturbation by the growth of the network. In the course of the initial spread of the excitation, the dynamics of the network is established, which ultimately determine the asymptotic behavior of the network.

In this report, we examine the spatiotemporal dynamics of a network of excitable nodes. We focus on an experimentally realizable network based on the photosensitive Belousov-Zhabotinsky system, ${ }^{15,16}$ in which the local excitability can be manipulated via light intensity. ${ }^{17}$ We first examine the spread of excitation across the network following a perturbation of the quiescent steady state. The conditions for sustained oscillations or the collapse of the dynamics to the steady state are then characterized. The nature of the oscillatory behavior in terms of frequency and phase locking of the underlying excitable dynamics is discussed. Finally, we examine how the network evolves as a consequence of the dynamical processes, and we identify the active subnetwork.

\section{NETWORK}

\section{A. Experimental system}

The experimental system consists of the photosensitive $\mathrm{BZ}$ reaction in which the trisbipyridine $\mathrm{Ru}(\mathrm{II})$ catalyst is immobilized in a thin layer $(0.3 \mathrm{~mm})$ of silica gel that is bathed in continuously refreshed catalyst-free solution. ${ }^{18} \mathrm{~A}$ video projector and video camera interfaced with a computer allow real-time feedback for perturbing the light sensitive medium and monitoring its response. The system is maintained in an 
excitable state with an illumination intensity of 8.82 $\times 10^{-3} \mathrm{~W} \mathrm{~m}^{-2}$. The gel area of $22 \mathrm{~mm} \times 22 \mathrm{~mm}$ is divided into a $26 \times 26$ cell array by the imposed light intensity, where each cell is made up of $10 \times 10$ grid points. The reaction mixture composition, $\left[\mathrm{BrO}_{3}^{-}\right]=0.552 \mathrm{M}, \quad[$ malonic acid] $=0.026 \mathrm{M}, \quad[$ bromomalonic acid $]=0.162 \mathrm{M}$, and $\left[\mathrm{H}_{2} \mathrm{SO}_{4}\right]$ $=0.489 \mathrm{M}$, was prepared so that the system is oscillatory in the dark state. Hence, a wave can be initiated at a cell by reducing the light intensity to zero in that cell.

\section{B. Model system}

Numerical studies were conducted using the Oregonator model $^{19,20}$ of the photosensitive BZ reaction ${ }^{16,21,22}$

$$
\begin{aligned}
& \frac{\partial u}{\partial t}=D_{u} \nabla^{2} u+\frac{1}{\epsilon}\left[u-u^{2}-(\phi+f v) \frac{u-q}{u+q}\right], \\
& \frac{\partial v}{\partial t}=u-v,
\end{aligned}
$$

where $u$ and $v$ are the dimensionless concentrations of $\mathrm{HBrO}_{2}$ and $\mathrm{Ru}(\mathrm{bpy})_{3}^{3+}, D_{u}$ is the diffusion coefficient of $\mathrm{HBrO}_{2}$, and $\phi$ represents the rate of photochemical bromide production due to the irradiation. The Euler method was used for the numerical simulations, with $d x=0.15$ and $d t=0.001$, and the parameters are $f=1.4, q=0.0002, \epsilon=0.01$, and $D_{u}$ $=1.0$, where $\epsilon$ and $q$ are scaling parameters and $f$ is an adjustable stoichiometric parameter. The network was comprised of a $50 \times 50$ cell array, with each cell made up of 19 $\times 19$ grid points. A wave could be initiated at a cell by setting $\phi=0$ at all grid points within the cell while the surrounding medium was maintained in the excitable state with $\phi=0.073$.

\section{Experimental and numerical methods}

Each cell in the square array of cells is considered to be a node in the static network. Local links arise naturally within the array from propagating reaction-diffusion waves, which emanate from initiation sites to propagate through surrounding cells. Directional nonlocal links are created by changing the excitability of a destination cell according to the state of a source cell. The resulting nonlocal links are assigned prior to commencing an experiment by randomly choosing a source cell $i$ and a destination cell $j$, where $i \neq j$. The threshold for activation of a link is determined by the excitation of $50 \%$ of the grid points in a source cell, which then triggers an increase in excitability in the destination cell. This increase in excitability may or may not give rise to the initiation of a new wave, depending on the dynamical state of the destination cell. The overall experiment is initiated by reducing the light intensity to zero in the center cell of the medium to initiate a wave propagating into the surrounding excitable cells. ${ }^{17}$

\section{NETWORK STRUCTURE}

The addressable excitable medium, with local and nonlocal excitations, has the structure of a static network with directed, weighted links. ${ }^{23}$ We consider the cells to be nodes

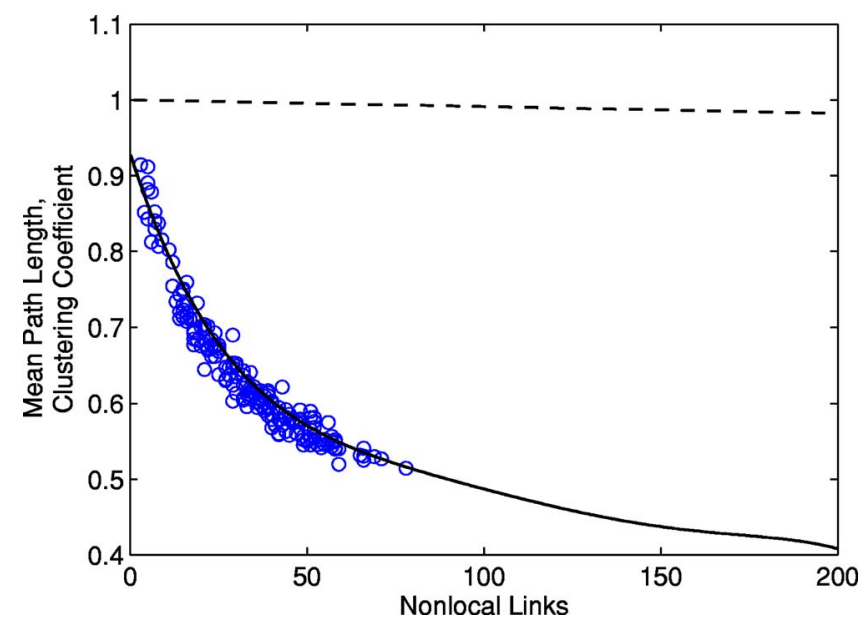

FIG. 1. The mean path length (solid line) and the clustering coefficient (dashed line) as a function of the number of randomly assigned nonlocal links. The values are normalized by the mean path length and the clustering coefficient, respectively, for the network with no random links. Circles show the normalized mean path length resulting from a reduction in the number of network links due to the dynamics of the excitable nodes (see Sec. IV D).

of the network, and the weighting of a link between nodes is determined by the time required for an excited node to give rise to an excitation at a node to which it is directly connected. For local links, this excitation time is governed by the geometrical distance between the nodes. The weighting for a nonlocal link is determined by the time between the excitation of the source node and the appearance of a wave at the destination node.

The underlying architecture of the network can be characterized according to the mean path length and the clustering coefficient. ${ }^{1,10}$ The mean path length is defined as the average minimum separation of two nodes within the network. The variation of the mean path length with the number of links is shown in Fig. 1. The clustering coefficient is defined as the average number of links between neighbors of an individual node divided by the total possible number of links between these neighbors. ${ }^{2,24}$ The statistical measures in Fig. 1 reveal a small-world network architecture as the number of possible links in the system is increased. At a low number of links, the mean path length and clustering coefficient reflect the underlying lattice network. At a high number of links, the mean path length scales as that of a random graph, ${ }^{25}$ while the clustering coefficient remains close to the value for a network with no random links. For an intermediate number of links, we see small path lengths and relatively large clustering coefficients typical of small-world networks. ${ }^{2}$

The initial spreading of excitation through the system can be characterized by recording the time each node is first excited following the central initiation of the experiment. The initial wave expands radially, and as source nodes are excited, new waves are initiated via links to destination nodes. Further initiations continue to occur until all of the nodes have been excited at least once. Figures 2(a) and 2(c) show typical configurations of the computational and experimental networks, respectively, where each node is shaded according to the time of excitation. The spreading of the 
(a)

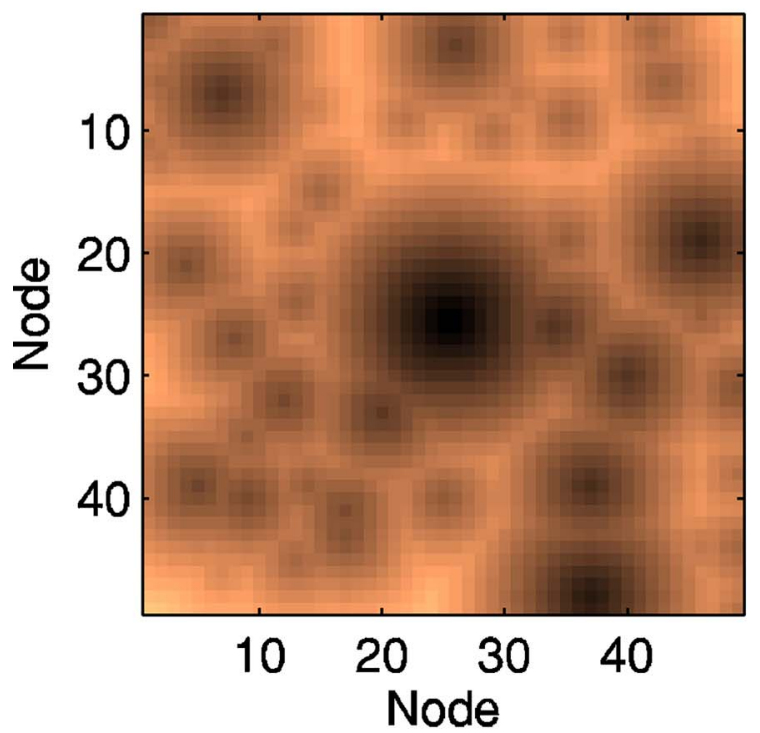

(c)
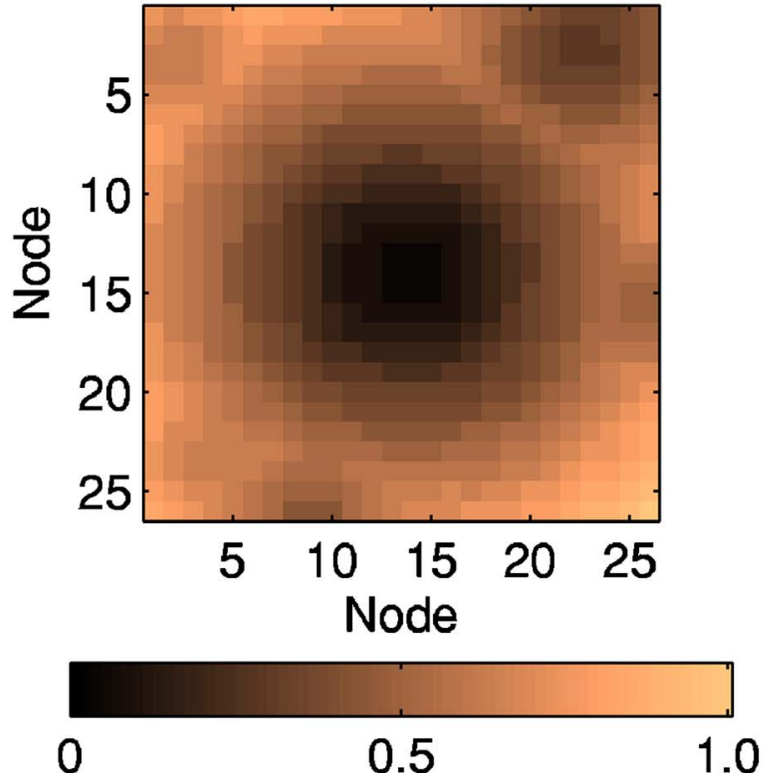

(b)

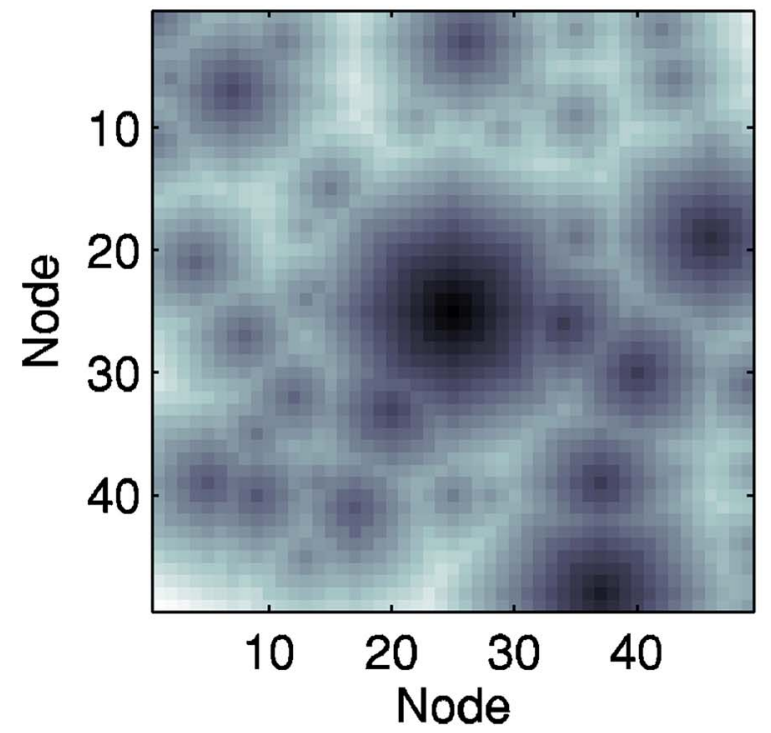

(d)
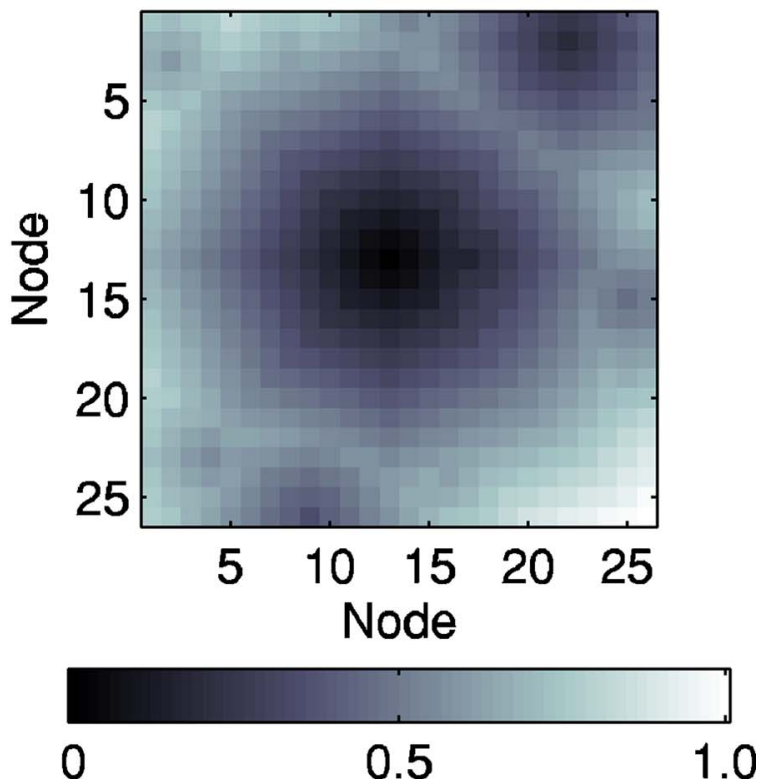

FIG. 2. (a) The normalized time of travel from the central wave initiation to each node. Numerical simulation for a system of $50 \times 50$ nodes and 100 nonlocal links. (b) The shortest path length from the central wave initiation to each node for the network in (a) calculated using Dijkstra's algorithm. A weighting of 0.4 is applied to the nonlocal links. (c) The normalized time of travel from the central initiation to each node for a $26 \times 26$ node experiment with 10 nonlocal links. (d) The shortest path length from the central initiation to each node for the network in (c) calculated using Dijkstra's algorithm. A weighting of 2.1 is applied to the nonlocal links.

central excitation is apparent as well as the subsequent nonlocal excitations.

The structural features of the network can be evaluated by examining the shortest distance between each node and the central node in the network, which can be determined using Dijkstra's algorithm. ${ }^{26}$ Figures 2(b) and 2(d) show networks with the same links as in the simulations and experiments in Figs. 2(a) and 2(c), where now each node is shaded according to its distance from the central node along the shortest pathway. The weighting of the links in Dijkstra's algorithm is determined by the time required for an excited node to excite the nodes to which it is directly connected.
The time required to excite the nearest neighbor nodes is used to normalize the times for the other links, and the weighting for the four nearest neighbor links is simply $w_{1}$ $=1$. We assume the time required to excite the next-nearest neighbor links is proportional to the geometrical distance between the nodes, and the weighting for these links is therefore $w_{2}=\sqrt{2}$. The initiation time for a nonlocal link is the time between excitation of the source node and the time of appearance of a wave at the destination node. The wave initiation time in the experimental system is significantly longer than in the model system, and different weightings for the 

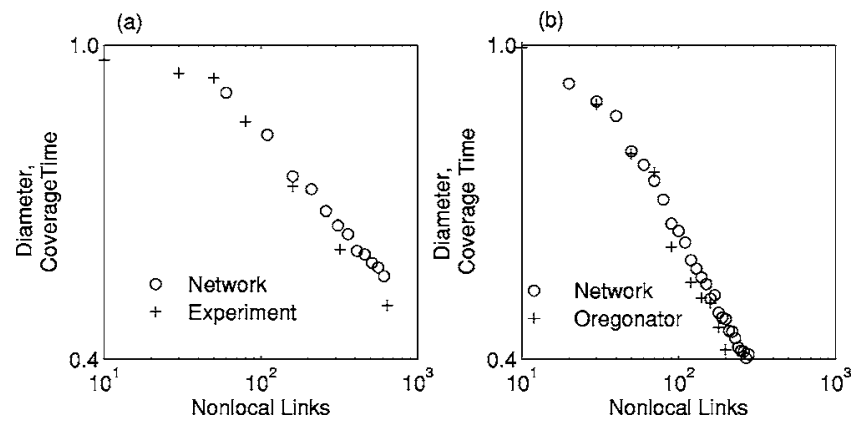

FIG. 3. (a) The normalized relative network diameter measured using Dijkstra's algorithm $(\bigcirc)$ and the normalized first coverage time $(+)$ as a function of the number of nonlocal links in an experimental $26 \times 26$ node system. Each point represents 10 network configurations. (b) The normalized relative network diameter $(\bigcirc)$ and the normalized first coverage time $(+)$ as a function of the number of nonlocal links in numerical simulations of a 50 $\times 50$ node system. Each point represents an average of 20 network configurations.

nonlocal links are therefore required in the corresponding Dijkstra's algorithm calculations. For the experimental and model networks, weightings of $w_{3, \text { exp }}=2.1$ and $w_{3, \text { num }}=0.4$ were used for the nonlocal links. The difference in the initiation times in the experiment and numerical networks, and hence the weightings of nonlocal links, is due to residual light intensity that is unavoidable in the experimental system as well as the nonquantitative nature of the model system.

The striking similarity between Figs. 2(a), 2(c), and 2(b), 2(d) indicates that the excitation propagates through the network via the shortest path between nodes. This result is related to the determination of optimal paths in a maze with propagating reaction-diffusion waves, ${ }^{27}$ as a maze is equivalent to a network of connected nodes. Thus, the minimal path length from the central initiation point to every node in the network is determined by the spread of excitation through the network and corresponds to the minimal path length determined by Dijkstra's algorithm.

The information in Figs. 2(a) and 2(c) also includes the first coverage time, which is the time taken for each node to be excited at least once following the central initiation. Similarly, the information in Figs. 2(b) and 2(d) includes the relative network diameter, which we define as the distance to the node that lies farthest from the central node measured along the network. ${ }^{2}$ Figure 3 shows the first coverage time and the relative network diameter as a function of the number of links for the experimental measurements and model system. The values of first coverage time and the relative network diameter are normalized with respect to the maximum values at zero link number. We see that the first coverage time dependence on the number of links is equivalent to the dependence of the relative network diameter calculated using Dijkstra's algorithm.

The measures shown in Figs. 1-3-the mean path, shortest path, and relative network diameter-demonstrate important features of the underlying network architecture and illustrate the ability to explore these features with the excitable reaction-diffusion system. However, even though an initial network structure is imposed on the system, the underlying dynamical processes give rise to the evolution of an effective
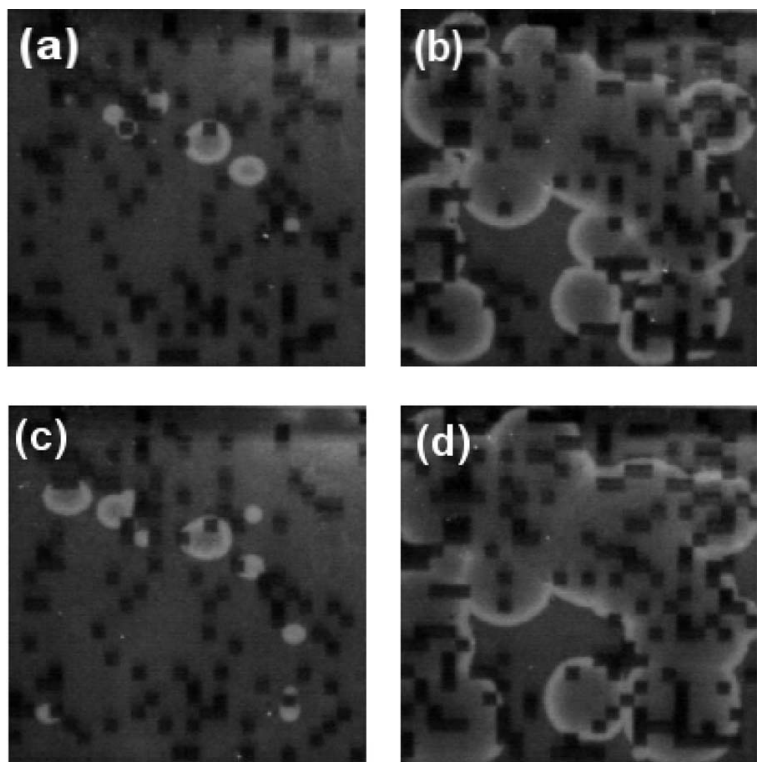

FIG. 4. Images of a typical experiment with a $26 \times 26$ node network and 300 nonlocal links. Dark squares represent activated destination nodes. Images were taken at $15 \mathrm{~s}$ intervals.

network. The nodes of the network are comprised of the excitable medium, and the grid points in each node exist in either an excitable, excited, or refractory state. Each part of the medium is maintained in the excitable state until it is excited locally by a propagating reaction-diffusion wave or nonlocally by a wave initiation at the destination node of an active link. Once excited, the medium locally undergoes an excitation cycle and cannot be excited again until it has relaxed back to the excitable state. Therefore, some nodes in the original network structure become unavailable for excitation as the result of local excitation cycles occurring throughout the medium. The measures that have been described in this section correspond primarily to the first excitation of the medium; we describe the dynamic evolution of the network in the next section.

\section{NETWORK DYNAMICS}

Figure 4 shows typical behavior of the experimental system in successive snapshots taken at $15.0 \mathrm{~s}$ intervals. The dark squares in each frame show the destination nodes of activated links, where the excitability is increased for possible wave initiation. The bright regions show the excited state of the reaction-diffusion waves, where the regions immediately behind are in the refractory state. Frames (a) and (c) were taken at an interval approximately equal to one period and show similar patterns of coverage, as do frames (b) and (d).

\section{A. Fractional coverage}

An instantaneous measure of the state of the system is given by the fractional coverage, defined as the fraction of the medium that is in the excited state at any time. Both the transient and asymptotic dynamics of the network are revealed as a function of time by this measure. Figure 5 shows the fractional coverage of a model system with 200 nonlocal 

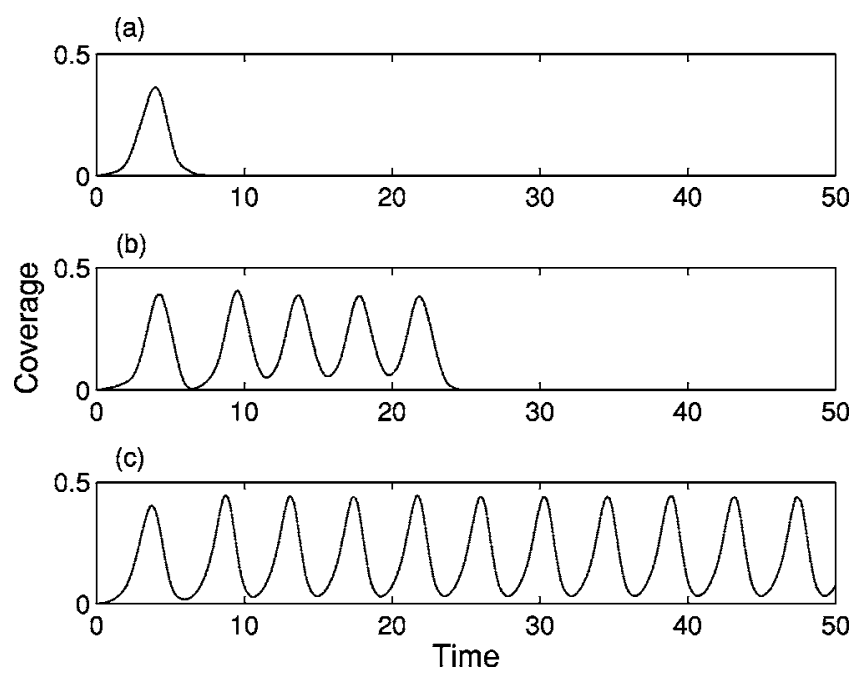

FIG. 5. Three types of behavior for the fractional coverage as a function of time: a single excitation (a), transient oscillations (b), and sustained oscillations (c). Simulations carried out with a $50 \times 50$ node network, with a different set of 200 nonlocal links in each case.

links, where three different types of behavior are observed depending on the initial conditions. The excitation may spread through the medium only once, with the system subsequently relaxing to the excitable steady state, as shown in Fig. 5(a), or sustained oscillations may be exhibited, as shown in Fig. 5(c). Occasionally, a number of transient oscillations are observed before the system collapses to the excitable steady state, as shown in Fig. 5(b).

As the number of nonlocal links is increased, it becomes more and more likely that the nodes of the network will each be excited only once. The dependence of the behavior on the number of links was investigated with the computational model to determine the probability of collapse to the steady state. Approximately 40 simulations with different initial conditions were carried out to determine the collapse probability for each link number shown in Fig. 6. We see that the

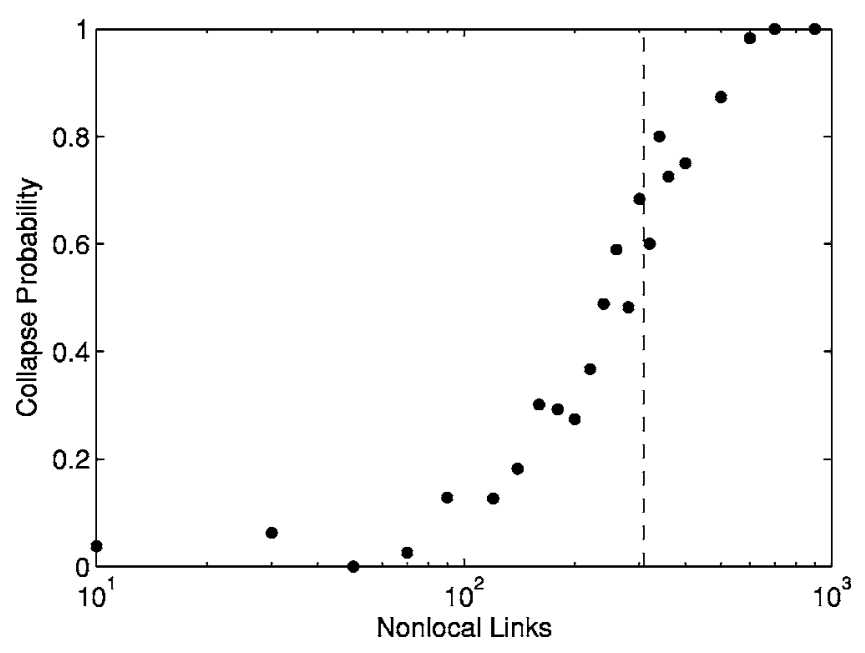

FIG. 6. Probability of collapse to the steady state as a function of the number of nonlocal links. Each point represents the average of approximately 40 configurations of the links. Simulations carried out with a 50 $\times 50$ node network. The dashed line corresponds to the number of links in which the first coverage time is equal to the period of the excitation cycle.
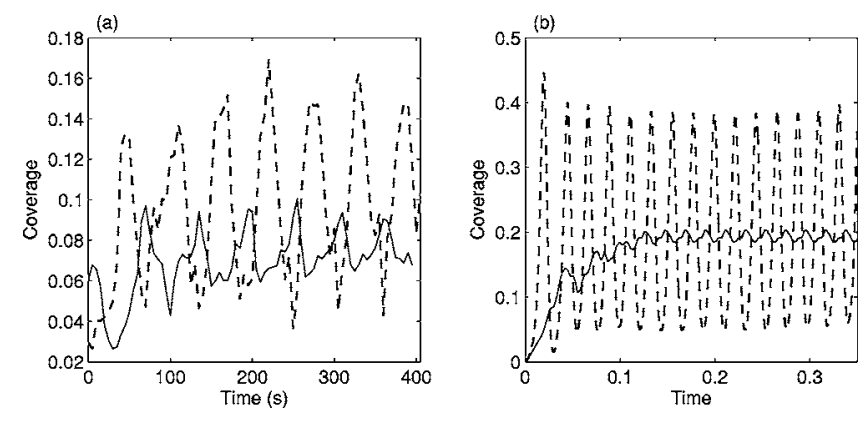

FIG. 7. (a) The fractional coverage as a function of time in experiments carried out on a $26 \times 26$ node network with 300 nonlocal links (dashed line) and 20 nonlocal links (solid line). (b) Fractional coverage as a function of time in simulations of a $50 \times 50$ node network with 200 nonlocal links (dashed line) and 10 nonlocal links (solid line).

probability of the system collapsing to the steady state increases with an increase in the number of nonlocal links. In a system with no nonlocal links, a single wave of excitation will propagate through the medium, with each node becoming excited once and remaining refractory for a period of time before relaxing to the excitable state. Secondary wave initiations can occur in a system with nonlocal links if the destination nodes are no longer in the refractory state when the link is activated. The transition to nonsustained oscillatory behavior is accompanied by a decrease in the first coverage time. The increase in collapse probability occurs when the first coverage time is approximately equal to the period of the excitation cycle. The dashed line in Fig. 6 indicates the number of links for which the first coverage time is equal to the excitation cycle period. Nonlocal links become ineffective at shorter first coverage times because the initiation of secondary waves at destination nodes is not possible when these nodes have not relaxed to the excitable state.

Typical sustained oscillations in the fractional coverage are shown in Fig. 7 for the experimental and model systems. We see in the experiments and simulations that the oscillation amplitude tends to increase as the number of nonlocal links is increased. The increase in amplitude corresponds to an increase in phase synchronization of the oscillatory behavior throughout the medium. Simple oscillations were the most common in both the experiments and the simulations, although complex oscillations were occasionally exhibited (which we exclude in our analyses). The oscillations in the experimental system tend to be less regular due to the inherent inhomogeneity of the system.

We note that collapse to the steady state was not observed in the experimental system. Possible explanations for this include the inherent inhomogeneities and the fact that the first coverage time is always longer than the period of the excitation cycle in the experimental system. Simulations were carried out to test these possibilities, in which a system with 200 links that exhibited collapse, as in Fig. 5(a), was modified. In one set of calculations, a delay in the wave initiation time was incorporated into the model to mimic the longer wave initiation time observed in the experiment. When the wave initiation time was increased by a factor of 5 , which was comparable to the wave initiation time in the experiment, sustained oscillations were observed. The delay 


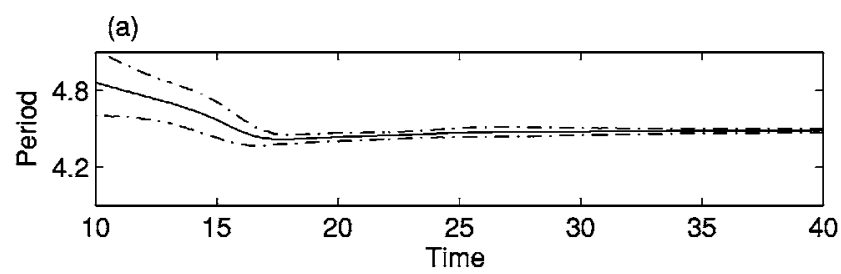

(b)
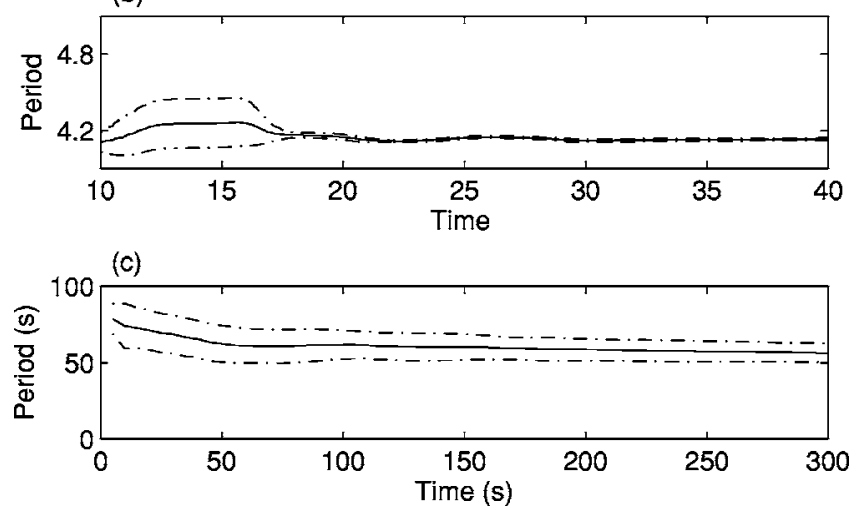

FIG. 8. The average period (solid line) and standard deviation (dashed lines) in simulation of a $50 \times 50$ node network with 30 nonlocal links (a) and 180 nonlocal links (b). The average period (solid line) and standard deviation (dashed lines) in experiment on a $26 \times 26$ node network with 300 links (c).

gives rise to an excitation cycle that is longer than the first coverage time, as observed in the experiment. Another set of calculations was carried out in which the value of $\phi$ was increased from 0.073 to 0.100 in one-half of the medium. Sustained oscillations were observed in the inhomogeneous system, while the homogeneous system collapsed to the steady state.

\section{B. Frequency synchronization}

Experiments and simulations were carried out to investigate the synchronization behavior of the nodes, where the state of each node was described by the average concentration of the inhibitor $\mathrm{Ru}(\mathrm{bpy})_{3}^{3+}$. The local intensity in the experimental images corresponds to the concentration of the inhibitor, and the local value of the variable $v$ corresponds to the inhibitor concentration in the simulations. The period of each node is then defined as the time between successive maxima in the corresponding time series. The period is never less than that of an excitation cycle; however, it may be longer if the node remains in the excitable state for extended times, and it can be infinite in the case of no subsequent excitations of a node.

Figure 8 shows the evolution of the average period of the nodes and the corresponding standard deviation as a function of time for simulations with 30 and 180 links and for an experiment with 300 links. As the network settles into its fractional coverage oscillation, the period of all of the nodes approaches a single value. Following a transient time, the standard deviation becomes very small in the simulations, shown in (a) and (b), as the average period of an individual node oscillation converges to the period of the coverage oscillation. Frequency synchronization is exhibited whenever sustained coverage oscillations occur, regardless of the number of nonlocal links, although the duration of the transient

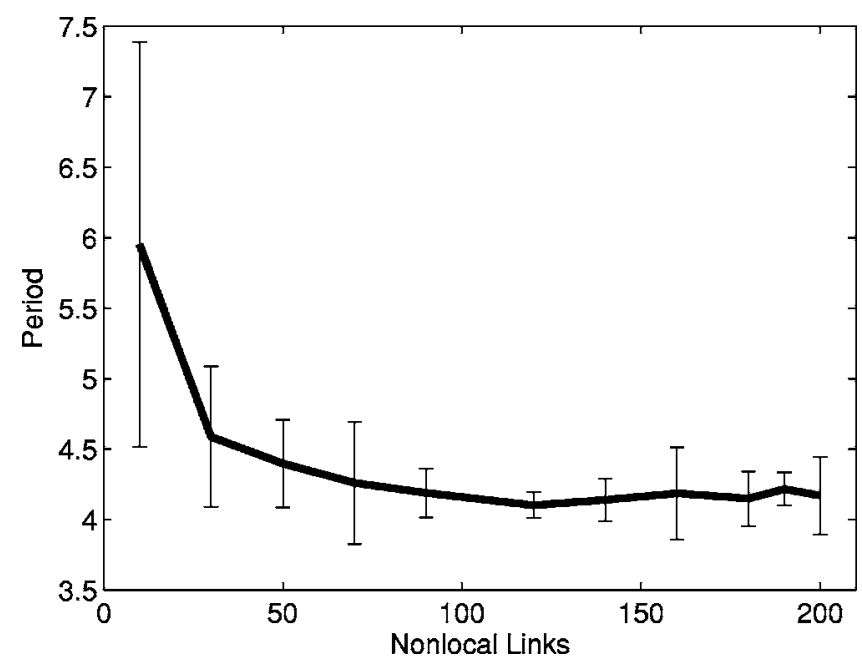

FIG. 9. The average period as a function of the number of nonlocal links in the model system. The error bars show the standard deviation corresponding to approximately 10 different configurations of the network.

time may depend strongly on the particular link configuration. We note that the larger standard deviation in the measurements shown in (c) is likely a reflection of the unavoidable inhomogeneities in the experimental system.

Frequency synchronization is the result of entrainment by the highest frequency network pacemaker, where a pacemaker is defined as a periodic source of excitation. In a network of excitable nodes, periodic sources of excitation arise when there are links with destination nodes behind waves that excite the source nodes of the links. Such an excitation loop forms a distributed network pacemaker, with a period defined by the time required for a wave to travel from the destination node to the source node plus the time for the nonlocal link to re-excite the destination node. These loops may have complicated structures, with multiple local and nonlocal links. An excitation loop will entrain all of the nodes in the network, provided that its period is shorter than that of any other excitation loop. These excitation loops lead to a dependence of the asymptotic period on the number of links, as shown in Fig. 9. We see that for greater than about 50 links, it is likely that there will be an excitation loop with the shortest possible period, which is governed by the period of the excitation cycle. Hence, as soon as a destination node behind a wave relaxes to the excitable state, it is excited by a link to an excited source node. For lower numbers of links, the asymptotic period also depends on the length of the shortest excitation loop, but now the period may be longer because destination cells behind waves may remain excitable for extended times. For few links, the period of the excitation loops becomes highly dependent on the network structure, and therefore a much larger standard deviation in the period is observed, as seen in Fig. 9.

\section{Phase synchronization}

We use a measure of the degree of phase synchronization given by the normalized vector sum of the phases, $R$ (Ref. 28), 


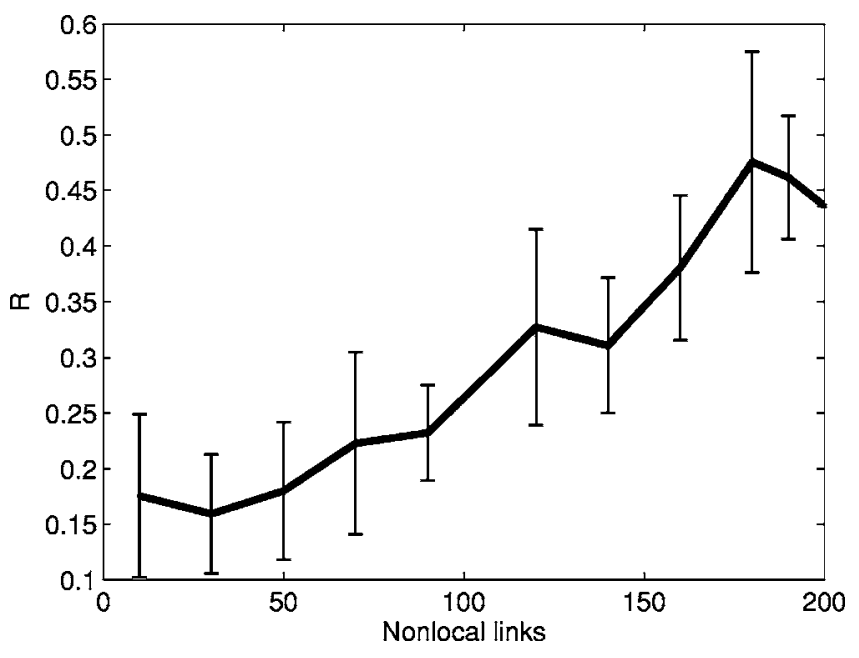

FIG. 10. Average value of $R$ calculated from Eq. (3) as a function of the number of nonlocal links in the simulation of a $50 \times 50$ node network. Values are for sustained oscillations in the fractional coverage.

$$
R=\frac{1}{N}\left|\sum_{j=1}^{N} e^{i \theta_{j}}\right| .
$$

A value of $R$ close to zero indicates that the phases of the individual oscillators are evenly distributed, while a value of $R$ close to unity indicates the oscillators are in phase. We do not expect to observe $R$ values close to unity, since sustained oscillations are not possible in a highly synchronized network of excitable nodes. Figure 10 shows that the phase synchronization increases as links are added to the network. A consequence of this synchronization is the increase in amplitude of the coverage oscillation with increasing numbers of links seen in Fig. 7.

\section{Active links}

The dynamics of the excitable medium making up the nodes of the network gives rise to a pruning process in which a subset of the links originally ascribed to the network becomes inactive, while the remaining active links account for the dynamical behavior of the system. The pruning occurs for particular spatiotemporal patterns because the destination nodes may be in the excited or refractory state and therefore cannot be excited. We define an active link as a nonlocal link that results in a successful initiation of a wave at the destination cell. The structure of the dynamical process that gives rise to oscillations in the coverage will now be examined according to the number and sequence of active links.

The number of active nonlocal links during regular coverage oscillations is shown as a function of the total number of nonlocal links in Fig. 11. A correlation is apparent, although there is an increasingly large spread in the values arising from the increase in possible network configurations. The average number of active links is a slightly decreasing fraction of the total as the number of random links increases. This slight falloff is apparently the result of a saturation effect, since the medium becomes more phase synchronized at higher link numbers. This gives rise to increasing numbers of

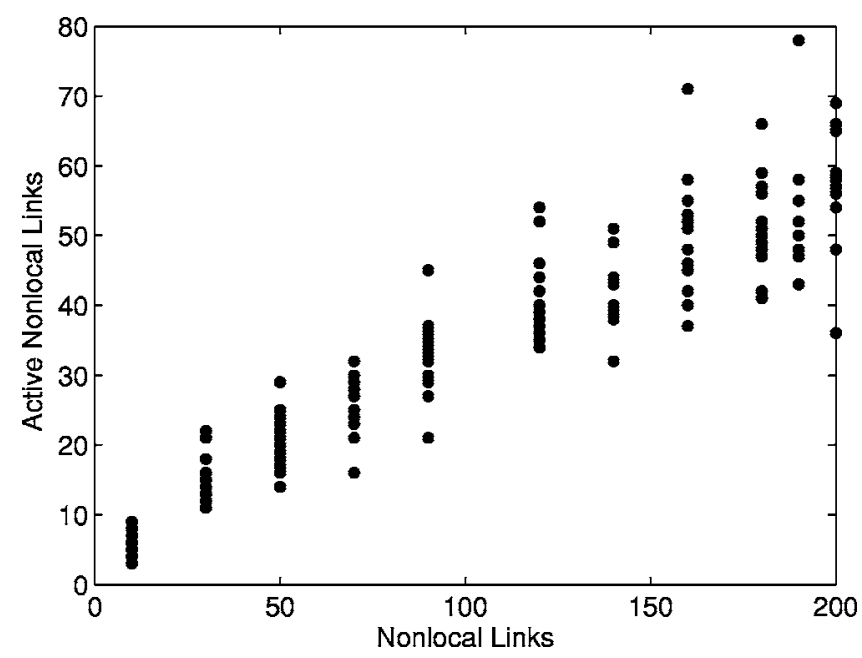

FIG. 11. The number of active nonlocal links as a function of the number of random nonlocal links in simulations of a $50 \times 50$ node network.

inactive links, as more source nodes are activated at coverage oscillation maxima when destination nodes are more likely to be unexcitable.

Further insights into the dynamics of the network can be gained from the sequence of active links. The repeating pattern of initiations that leads to the overall oscillations can be examined in terms of the sequence in which the links are activated. Destination nodes of active links are labeled according to the order of their excitations during the asymptotic coverage oscillations. The active link with its destination node closest to the center of the medium is chosen to be link 1, and the subsequent active links are successively numbered at their destination nodes. This leads to a sawtooth profile in the order of destination node excitation, increasing from 1 to the total number of active links, when plotted as a function of time. This sequence of initiations is repeated once per coverage oscillation, as shown in Fig. 12(a). The sequence of active links may be stable or unstable to particular perturbations, and these stability properties are demonstrated in Fig. 12. Active link 6 is removed for several oscillatory cycles from the previously stable sequence in Fig. 12(b). We see that following a transient period, the system finds a new cycle of sequential active links. Active links that were not involved in the original sequence are numbered according to their order of first occurrence during the transient. In Fig. 12(c), active link 4 is removed for several oscillatory cycles. We see that the sequence is stable to this perturbation, as it quickly returns to the original cycle. The experimental system exhibits a similar sequencing of active links over successive coverage oscillations, as shown in Fig. 13. We see that there is a spontaneous shifting of the active link basis for the experimental coverage oscillation during the transient period before the system relaxes to its asymptotic sequence.

The impact of the underlying dynamics on the network structure can be seen by replotting the effective mean path length as a function of the number of links, where the values are determined by including only the active links. We see in Fig. 1 that the effective mean path length scales in the same 

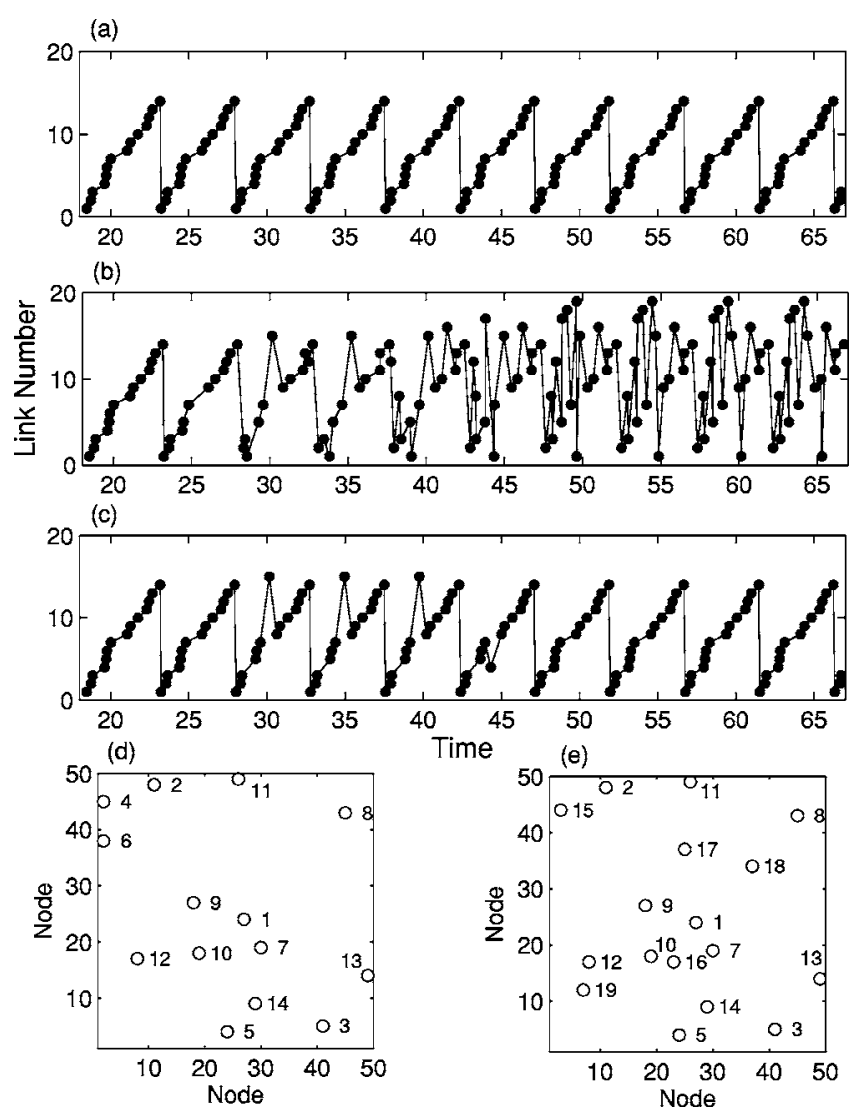

FIG. 12. (a) Sequence of node excitation for 30 nonlocal links in the simulation of a $50 \times 50$ node network. (b) Sequence of node excitation with the removal of active link 6 from $t=24.4$ to $t=40$.0. A new sequence is displayed that persists after link 6 is replaced. (c) Sequence of node excitation with the removal of active link 4 from $t=24.4$ to $t=40.0$. The original sequence is displayed after the link is restored. (d),(e) Locations of the active links plotted in the sequence shown in (a),(b).

manner as the equivalent sized random network mean path length. The underlying excitable dynamics has therefore selected a dynamically equivalent subnetwork from the initial network of local and nonlocal links.

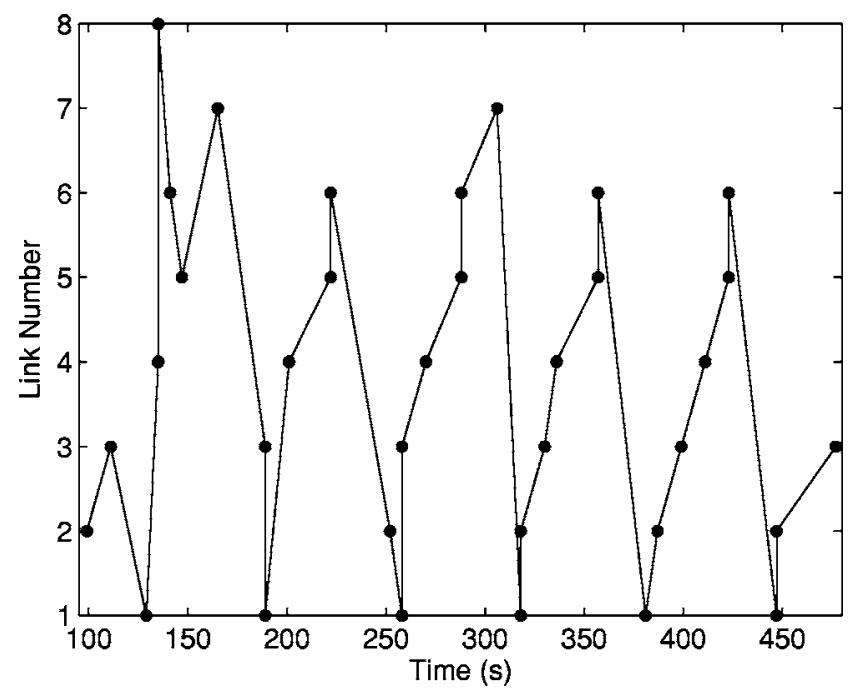

FIG. 13. The active link sequence for a network with 10 links determined in an experiment on a $26 \times 26$ node network. Links 7 and 8 are spontaneously eliminated as the network evolves to a periodic sequence.

\section{DISCUSSION}

The excitation spreads through the network from the initial central wave excitation to reach each node via the shortest path. We find that the time required for the final node to become excited varies in the same manner that the relative diameter of the network varies with the number of links. Oscillatory asymptotic behavior is more likely for networks with low numbers of links, while collapse to the excitable steady state is more likely for networks with high numbers of links. The sustained oscillations in the fractional coverage arise from the entrainment of the individual nodes of the network to the highest frequency network pacemaker. The associated phase and frequency synchronization of the nodes is a consequence of the connectivity of the underlying network and the ability of each excitable node to respond to the highest frequency perturbation. We note that simple oscillatory behavior was observed in almost all of our experiments and simulations, and higher-order periodicities in the coverage oscillations were observed in only a handful of cases.

Insights into the network pacemaker can be gained by considering an extreme example. We imagine a network with only a single nonlocal link, with the source node an appropriate distance from the destination node at the center of the medium. The resulting spatiotemporal pattern will consist of expanding circular waves, with a frequency dependent on the distance of the source node from the destination node. The coverage oscillation simply corresponds to the increase in excitation area as the wave expands and eventually leaves the medium, which gives rise to a small amplitude oscillation on repeated wave initiations at the destination node. We have seen with increasing numbers of nonlocal links that the average amplitude of the coverage oscillation increases, indicating that a higher fraction of the nodes are simultaneously excited with increasing link connectivity.

The dynamical features of the excitable nodes give rise to a pruning process, where a significant fraction of the links becomes inactive and a subnetwork of active links determines the asymptotic dynamics. We have seen that the link sequence is stable with respect to some link perturbations and unstable with respect to others. The selection of a subnetwork by the collective dynamics of the excitable nodes has features akin to the selection of subnetworks or network motifs in genetic networks and food webs. ${ }^{29}$

The network of excitable nodes in the photosensitive BZ system offers a means to demonstrate synchronization in an experimental system with local and nonlocal links. The transition to synchronized behavior, through frequency locking and an increase in the order parameter $R$, is similar to that seen in other networks of oscillatory ${ }^{10,11}$ and excitable $e^{6,12,14}$ nodes. We note that similar synchronization also has been found in a system of globally coupled electrochemical oscillators. ${ }^{30}$ While understanding the synchronization of a network of oscillatory or excitable nodes is essential for a characterization of the system dynamics, other features such as network growth and the relationship between network architecture and node dynamics yield insights into the spatiotemporal behavior. We note that the term network growth has several meanings depending upon the context. In describing network architectures, it typically refers to the addi- 
tion of nodes to an existing network based upon a specified attachment rule. ${ }^{2}$ In the work described here, the network develops from an existing network architecture according to the dynamical processes occurring in the network. We have seen how the initial spread of activity, following an initiation stimulus, traverses the network via the optimal shortest path. The development of the asymptotic behavior is governed by a pruning process in which an active subnetwork is selected according to the intrinsic time scale of the node dynamics and the architecture of the network.

\section{ACKNOWLEDGMENTS}

We thank the National Science Foundation (CHE0415392) and the W. M. Keck Foundation for supporting this research. K.S. thanks the Alexander von Humboldt Foundation for supporting research visits to the Fritz Haber Institute, Berlin. A.J.S. thanks the National Physical Science Consortium for support.

${ }^{1}$ D. J. Watts and S. H. Strogatz, Nature (London) 393, 440 (1998).

${ }^{2}$ R. Albert and A. L. Barabási, Rev. Mod. Phys. 74, 47 (2002).

${ }^{3}$ M. Chavez, D. U. Hwang, A. Amann, H. G. E. Hentschel, and S. Boccaletti, Phys. Rev. Lett. 94, 218701 (2005).

${ }^{4}$ L. G. Morelli, H. A. Cerdeira, and D. H. Zanette, Eur. Phys. J. B 43, 243 (2005).

${ }^{5}$ O. Sporns and G. Tononi, Complexity 7, 28 (2002).

${ }^{6}$ A. Roxin, H. Riecke, and S. A. Solla, Phys. Rev. Lett. 92, 198101 (2004).

${ }^{7}$ J. Pham, K. Pakdaman, and J. F. Vibert, Phys. Rev. E 58, 3610 (1998).
${ }^{8}$ J. Eggermont, Neurosci. Biobehav Rev. 22, 440 (1998).

${ }^{9}$ K. Kori and A. S. Mikhailov, Phys. Rev. Lett. 93, 254101 (2004).

${ }^{10}$ H. Hong, M. Y. Choi, and J. K. Beom, Phys. Rev. E 65, 026139 (2002).

${ }^{11}$ M. Barahona and L. M. Pecora, Phys. Rev. Lett. 89, 054101 (2002).

${ }^{12}$ P. Jung and P. C. Gailey, Ann. Phys. 9, 697 (2000).

${ }^{13}$ N. Masuda and K. Aihara, Biol. Cybern. 90, 302 (2004).

${ }^{14}$ M. Kuperman and G. Abramson, Phys. Rev. Lett. 86, 2909 (2001).

${ }^{15}$ A. N. Zaikin and A. M. Zhabotinsky, Nature (London) 255, 553 (1970).

${ }^{16}$ L. Kuhnert, Nature (London) 319, 393 (1986).

${ }^{17}$ M. Tinsley, J. Cui, F. Chirila, A. Taylor, S. Zhong, and K. Showalter, Phys. Rev. Lett. 95, 038306 (2005).

${ }^{18}$ T. Sakurai, E. Mihaliuk, F. Chirila, and K. Showalter, Science 296, 2009 (2002).

${ }^{19}$ R. J. Field and R. M. Noyes, J. Chem. Phys. 60, 877 (1974).

${ }^{20}$ J. J. Tyson and P. C. Fife, J. Chem. Phys. 94, 2224 (1990).

${ }^{21}$ H. J. Krug, L. Pohlmann, and L. Kuhnert, J. Phys. Chem. 94, 4862 (1990).

${ }^{22}$ S. Kádár, T. Amemiya, and K. Showalter, J. Phys. Chem. 101, 8200 (1997).

${ }^{23}$ M. Newman and D. Watts, Phys. Rev. E 60, 7332 (1999).

${ }^{24} \mathrm{We}$ define two neighbors to be locally connected if the distance between them is less than or equal to $\sqrt{2}$. All local links are considered to be bidirectional. The clustering coefficient $C$ for the network can be written as a function of the number of random links $r, C=\frac{3}{7}-(r / N)(2 / 21)$, where $N$ is the number of network nodes. In the numerical simulation, $N=2500$ and $r \leqslant 1000$, while in the experiment, $N=676$ and $r \leqslant 300$.

${ }^{25}$ D. J. Watts, Small Worlds (Princeton University Press, Princeton, 1999).

${ }^{26}$ E. W. Dijkstra, Numer. Math. 1, 269 (1959).

${ }^{27}$ O. Steinbock, Á. Tóth, and K. Showalter, Science 267, 868 (1995).

${ }^{28} \mathrm{Y}$. Kuramoto, Chemical Oscillations, Waves, and Turbulence (SpringerVerlag, New York, 1984).

${ }^{29}$ R. Milo, S. Shen-Orr, S. Itzkovitz, N. Kashtan, D. Chklovskii, and U. Alon, Science 298, 824 (2002).

${ }^{30}$ I. Kiss, Y. Zhai, and J. Hudson, Science 296, 1676 (2002). 